\title{
Reafference learning in the presence of exafference
}

\author{
JOHN C. HAY \\ University of Wisconsin, Milwaukee, Wisconsin 53201
}

\begin{abstract}
Reafference learning has been demonstrated most clearly in the case of position-constancy adaptation in which the only stimulus change is caused by the subject's own movement. The present study used the more ecologically representative training situation in which only part of the stimulus change is caused by the subject (reafference), while part of it is caused by an independent source (exafference). The exafference varied the space relation between subject movement and optical movement or the time relation between those two. In both cases, reafference learning was not affected by the exafference, and the subject's varied training experience resulted in a fixed expected optical movement and a fixed expected time lag.
\end{abstract}

In reafference learning, a person learns to expect a certain stimulus change whenever he makes a certain movement (Held, 1961; Welch, 1978). For example, if the person is trained with a diagonal optical movement paired with vertical head turning, he/she comes to require a portion of that diagonal optical movement for an object to appear stationary during that head movement. This specific phenomenon is called position constancy adaptation and is the best exemplification of reafference learning (Hay, 1968, 1974; Shebilske, 1977). However, the ecological representativeness of the usual laboratory procedure is doubtful. In the real world, other things besides the observer move, and hence there will be a less than perfect correlation between subject movement and optical movement. Externally caused components of stimulus change are called exafference, in contrast with the subject-caused component called reafference (Holst, 1954). The question of the present study is: What happens to reafference learning when exafference is present, when there is an imperfect correlation between the person's movement and stimulus change?

The strategy of this study is to see how two of the known properties of position constancy adaptation change when exafference is present during the training situation. Hay (1968) made such a preliminary investigation. Hay and Goldsmith (1973) have found that the adaptation state has two components: an expected optical motion (EOM) and an expected time lag (ETL) between the head movement and the

\footnotetext{
This research was supported by National Science Foundation Grant GB-33449 to the University of Wisconsin-Milwaukee, with the author as principal investigator. A qualitative summary of the results was given in Hay (1974) and was referred to in Welch (1978).
}

optical movement. We shall therefore introduce exafference into both of the corresponding components of the training situation: the optical-motion component (Experiment 1) and the time-lag component (Experiment 2).

\section{EXPERIMENT 1}

A luminous spot in an otherwise dark room is made to undergo horizontal oscillation when the subject nods his head up and down. The resulting diagonal optical motion (OM) relative to the head constitutes the training reafference. Exafference is added to this in the form of a horizontal, vertical, or combined horizontal-and-vertical component of spot movement. The amplitude of the exafferent movement is equal to that of the reafference; its frequency is randomly varied, while the headmovement frequency is fixed. The result is that, at any given moment, the spot undergoes a variable motion relative to the head but, over time, the average spot movement during head movement is equal to the training reafference.

\section{Method}

Subjects. Twenty men and 20 women, ranging from 18 to 29 years of age were recruited from psychology classes and were assigned randomly, within sexes, to four training conditions, so there were five men and five women in each. They were paid $\$ 2.00$ for their work, which usually took about $40 \mathrm{~min}$.

Apparatus. The apparatus of Hay and Goldsmith (1973) was used, with the addition of two sine-wave generators for the exafferent motion. The general nature of the apparatus will be described here.

The subject nods his head up and down on a fixed axis from $15 \mathrm{deg}$ above to $15 \mathrm{deg}$ below his preferred head-level position, at a $1.5-\mathrm{Hz}$ frequency paced by an audible metronome. He keeps his gaze trained on a $.5-\mathrm{cm}$-diam luminous spot in an otherwise dark room. During training, the spot undergoes a horizontal 
reafferent motion of $7.5 \mathrm{~mm}$ for every degree of vertical head movement. This is termed a 7.5-mm/deg HM:OM reafference. If the spot moves right when the head turns up, the reafference is termed positive; if the converse, it is termed negative. During the position constancy test, the reafference gain factor is controlled by the subject and is set by him to a value that makes the spot appear stationary during his head-nodding. Adaptation to a positive training reafference would consist of a positive pretest to posttest shift in the position constancy settings.

A dental wax biteboard attached to a rotational axis at the left of the subject's neck controls and measures the subject's head movement. A potentiometer on the axis produces a voltage analog of head position, and this is digitized once every millisecond by a PDP $8 / \mathrm{L}$ computer. Head position values are stored successively in a 1,000-position closed loop list in the computer memory. Head position values are retrieved from this list with a separation between storage and retrieval list pointers equal to the desired time lag in milliseconds. In this experiment, the time lag was set to the minimum possible with the apparatus, $1 \mathrm{msec}$. Retrieved head position values are multiplied by the reafference gain factor. During training, this is set by the experimenter; during testing, the subject's adjustment of a 10-turn potentiometer determines the gain factor. The multiplied head position value is reconverted to an analog voltage and delivered to one side of the differential horizontal amplifier of a cathoderay oscilloscope (CRO). The CRO trace is imaged on a $1-\mathrm{m}$ square rear-projection screen by a lens and is viewed by the subject at a distance of $1 \mathrm{~m}$ in a light-tight tunnel. The $.5-\mathrm{cm}$ luminous spot had an intensity of $.13 \mathrm{~cd} / \mathrm{m}^{2}$; the background screen intensity was $.0034 \mathrm{~cd} / \mathrm{m}^{2}$. Ten minutes of dark adaptation to this illumination was afforded by the pretraining phase of the experiment (see Procedure section).

Exafference was added to the diagonal OM of the reafference by means of two Hewlett-Packard Type 3300A sine-wave generators (SWGs). The first SWG drove the vertical amplifier of the CRO. Its signal was thus added in vector fashion to the horizontal amplifier's head movement signal. The result was that, during head movement, the luminous spot moved through a varying two-dimensional curved path, approximating a variable Lissajous pattern. This constituted the vertical exafference training condition. The second SWG was connected to the other side of the horizontal amplifier's differential input; its signal was thus added algebraically to the head movement signal, causing the horizontal motion of the spot to vary in direction and speed relative to the head. This constituted the horizontal exafference training condition. The amplitude of both SWGs was set to equal the full amplitude of the reafferent signal (i.e., $225 \mathrm{~mm}$ of spot movement on the screen). The frequency of the SWGs was independently varied by the computer, using a stored random number table that was used to produce new analog voltage levels once every half second to each of the voltage-controlled-frequency inputs of the SWGs. The result was that the exafferent frequencies changed once every $.5 \mathrm{sec}$ between limits of 1 and $2 \mathrm{~Hz}$, using 18 equally spaced steps.

Procedure. All subjects were pretrained to nod their heads at the $1.5-\mathrm{Hz}$ frequency set by the metronome, and they were given 10 practice trials on the position constancy test. Only persons who set the gain to within $\pm 1 \mathrm{~mm}$ of spot movement per degree of head movement ( $\pm 1 \mathrm{~mm} / \mathrm{deg}$ of HM:OM reafference) were admitted to the set of 40 subjects; six persons were rejected by this criterion.

Ten pretest trials were then given, followed by 1030 -sec training periods under one of the following four training conditions. In all training conditions, half the subjects experienced a $+7.5-\mathrm{mm} / \mathrm{deg} \mathrm{HM}: \mathrm{OM}$ reafference, while the others experienced a $-7.5-\mathrm{mm} / \mathrm{deg}$ HM:OM reafference. This meant that during the 30-deg up-and-down head nodding, with its $1.5-\mathrm{Hz}$ paced frequency, the subjects experienced a $225-\mathrm{mm}$ left-and-right component of optical movement, in phase with the head movement.
The four training conditions were as follows- (1) control training condition: no exafference; (2) vertical exafference training condition: $225 \mathrm{~mm}$ of vertical exafferent optical motion, with a frequency varying from 1 to $2 \mathrm{~Hz}$, added vectorwise to the reafferent motion; (3) horizontal exafference training condition: $225 \mathrm{~mm}$ of horizontal exafferent optical motion, with a frequency varying from 1 to $2 \mathrm{~Hz}$, added algebraically to the reafferent motion; and (4) combined vertical and horizontal exafference training condition: $225 \mathrm{~mm}$ of horizontal and $225 \mathrm{~mm}$ of vertical exafferent optical motion, each component having an independently varying frequency from 1 to $2 \mathrm{~Hz}$, added to the reafference motion.

After each $30 \mathrm{sec}$ of training, a single posttest of position constancy was given. After the full $5 \mathrm{~min}$ of training, 10 final posttests were given. It should be noted that no exafference was present during the position constancy tests.

\section{Results}

Figure 1 shows a sample recording of the effect of horizontal exafference during training. A more quantitative measure of the effect of exafference on the subjects' training experience was obtained by a correlation analysis of 9-sec segments taken from each of the 10 subjects in Training Condition 3 (horizontal exafference). Three properties of HM and $\mathrm{OM}$ were correlated, and the resulting values (with $99 \%$ confidence limits) were as follows. For the correlation of optical position $(\mathrm{O})$ and head position $(\mathrm{H}), \mathrm{r}=+.66 \pm .12$. For the correlation of optical velocity $(\dot{O})$ and head velocity $(\dot{H}), r=+.64$ \pm .15 . For the correlation of optical acceleration (Ö) and head acceleration $(\dot{\mathrm{H}}), \mathrm{r}=+.49 \pm .20$.

The principal measure of the effect of training
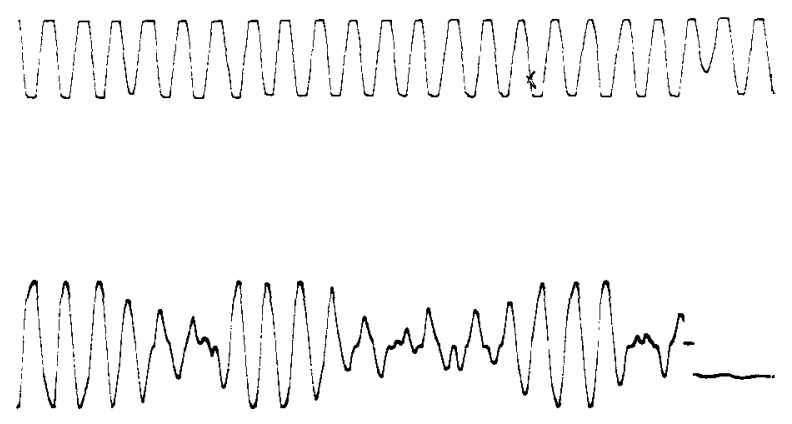

Figure 1. Sample recording of head movement and optical movement during reafference training with horizontal exafference. The top record is of the head movement, up and down between stops set to 15 deg above and below the horizontal, paced by a metronome at $1.5 \mathrm{~Hz}$. The lower record is of the horizontal movement of the luminous spot, left (down on the record) and right (up on the record), with a maximum amplitude of about $450 \mathrm{~cm}$ on the screen, viewed from a distance of $1 \mathrm{~m}$. For this particular subject, the training reafference was $-7.5 \mathrm{~mm} / \mathrm{deg}$. The time line at the bottom marks seconds. The beginning of an automatically programmed position constancy test is shown at the far right of the record, in which the subject continues nodding his head while adjusting the spot movement to appear stationary. 
Table 1

Position Constancy Judgments in Experiment 1

\begin{tabular}{|c|c|c|c|c|c|c|}
\hline \multirow{2}{*}{$\begin{array}{c}\text { Exafference in } \\
\text { Training }\end{array}$} & \multicolumn{2}{|c|}{ Pretest } & \multicolumn{2}{|c|}{ Posttest } & \multicolumn{2}{|c|}{ Change in Mean } \\
\hline & Mean & \pm & Mean & \pm & Mean & \pm \\
\hline None & -.07 & .30 & +.48 & .36 & +.55 & .39 \\
\hline Vertical & +.02 & .18 & +.53 & .44 & +.50 & .47 \\
\hline Horizontal & -.08 & .09 & +.28 & .30 & +.36 & .33 \\
\hline Both & -.11 & .24 & +.32 & .22 & +.32 & .23 \\
\hline
\end{tabular}

Note-The measures are in terms of the amount of horizontal optical motion (in millimeters) per degree of vertical head motion. Positive values indicate optical motion in the direction of that during training. The values are based on 10 pretests and 10 posttests for each subject, there being 10 different subjects in each training condition. $\pm=99 \%$ confidence limits.

Table 2

Variability of Position Constancy Judgments in Experiment 1

\begin{tabular}{|c|c|c|c|c|c|c|}
\hline \multirow{2}{*}{$\begin{array}{c}\text { Exafference in } \\
\text { Training }\end{array}$} & \multicolumn{2}{|c|}{ Pretest } & \multicolumn{2}{|c|}{ Posttest } & \multicolumn{2}{|c|}{ Change in SD } \\
\hline & SD & \pm & SD & \pm & Change & \pm \\
\hline None & .40 & .15 & .42 & .22 & .02 & .18 \\
\hline Vertical & .40 & .26 & .46 & .26 & .06 & .25 \\
\hline Horizontal & .41 & .20 & .44 & .21 & .03 & .36 \\
\hline Both & .42 & .17 & .46 & .25 & .04 & .20 \\
\hline
\end{tabular}

Note-The measures are in terms of the amount of horizontal optical motion (in millimeters) per degree of vertical head motion. They are based on 10 pretests and 10 posttests for each subject, there being 10 different subjects in each training condition. $\pm=99 \%$ confidence limits.

was the change in the mean position-constancy judgments from pretest to posttest. These findings are given in Table 1. All four training conditions show adaptive changes significant at the .01 level. An analysis of variance showed no differences among training conditions. The only significant effect was that of the pretest-posttest factor $[F(1,36)=48.755804$, $\mathrm{p}<.001]$. Although the mean adaptations were smaller in those training conditions having horizontal exafference (see Table 1), this factor did not have a statistically significant effect. For its interaction with the pretest-posttest factor $[F(1,36)=1.424813$, $\mathrm{p}>$.05].

Table 2 shows the variable errors of the pretest and posttest position constancy judgments. These show no significant changes due to training. An analysis of variance found no pretest-posttest difference $[F(1,36)=1.267516, p>.20]$.

The nine position-constancy tests given singly after each 30-sec segment of training also showed only a significant main effect of training $[F(10,360)=$ $5.129140, \mathrm{p}<.001]$.

\section{Discussion}

These results show no effect of exafferent motion on the expected optical motion component of reafference learning. They thereby confirm and extend the findings of Hay (1968), who obtained sim- ilar results in a vertical exafference training condition. After $5 \mathrm{~min}$ of training, the same level of adaptation to a diagonal optical motion during vertical head movement was found, whether the subject had been trained with the pure diagonal optical motion or with a widely varying curved optical motion.

The expected optical motion seems to be learned independently of the subject's conscious awareness of the connection between his head movement and the luminous spot's movement. In a debriefing after the experiment, none of the 30 subjects in the exafference training conditions could give a correct description of the effect of head movement on the spot. Five of the 10 subjects in the no-exafference control condition could do so. But this variation in consciousness of the reafference had no apparent effect on the reafference learning.

\section{EXPERIMENT 2}

An exafference was introduced in the time relation between head movement and optical movement during training: The time lag varied from 5 to $225 \mathrm{msec}$, with a mean $(115 \mathrm{msec})$ similar to the one $(150 \mathrm{msec})$ subjects were able to learn in Experiment 1 of Hay and Goldsmith (1973). The tests for adaptation followed the strategy of that earlier study: The subjects adjusted the gain factor relating head and spot motion under various time-lag conditions. If the adaptation has a time-lag characteristic, the adaptation should be measurable only by a test condition having that time lag.

\section{Method}

Subjects. Twenty women and 12 men, between 17 and 25 years of age, were recruited from the same population and were paid the same amount. They were all given the same reafference training condition and were divided equally among four testing conditions.

Apparatus. The apparatus of Hay and Goldsmith (1973) was again used, modified so that the adjustable time lag of that apparatus varied randomly during training. The mean time lag was set to $115 \mathrm{msec}$; the digitized voltage from a triangular-wave generator (WaveTek 131A) was used to modulate this time lag by an additive factor of $\pm 110 \mathrm{msec}$. This provided the exafferent time-lag component of training. The PDP 8/L sampled the waveform once every half second, so that the time lag changed twice per second. The time lag was monitored with a time-interval meter (Hickock DP210).

During the position constancy tests, the time lag was set to a constant value by the experimenter, and the subject adjusted the gain-control knob until the apparent horizontal motion of the spot was minimized, as in Experiment 1.

Procedure. Subjects were given the same pretraining on the position constancy test as in Experiment 1, using the time lag appropriate to their test condition.

The reafference training for all subjects involved an $11.5-\mathrm{mm} / \mathrm{deg}$ HM:OM reafference, larger than in Experiment 1 since the reafferent motion did not need to fit into the projection screen along with an exafferent motion component. Half the subjects in each group were trained with a +11.5-HM:OM reafference, while the others were trained with a $-11.5-\mathrm{HM}: \mathrm{OM}$ reafference. 
The variable 5- to 225-msec time lag was present throughout the training period, which again lasted $5 \mathrm{~min}$. No interspersed position constancy tests were given during training.

Ten pretests and 10 posttests were given to each subject, under one of the following four time-lag conditions. (1) Test Condition 1: fixed time lag of $115 \mathrm{msec}$ between head movement and any corresponding horizontal spot movement selected by the subject with his gain-control knob. This fixed time lag equaled the mean of the variable training time lag. (2) Test Condition 2: fixed time lag of $5 \mathrm{msec}$, equal to the minimum during training. (3) Test Condition 3: fixed time lag of $225 \mathrm{msec}$, equal to the maximum during training. (4) Test Condition 4: varying time lag of from 5 to $225 \mathrm{msec}$, the same as during training.

It should be noted that only in Test Condition 4 could the subject obtain a stimulus that would match, in both its optical motion and time-lag properties, the training condition. In the other test conditions, the time-lag component was fixed and could match only the mean, minimum, or maximum of the training time lag, depending on the condition.

\section{Results}

The effects of the variable time lag during training were measured by a cross-correlation analysis of 9-sec samples taken from all 32 subjects (Table 3). These indicate that the maximum measured correlation was at a time lag of $100 \mathrm{msec}$, the closest measured time lag to the mean set by the computer $(115 \mathrm{msec})$. It should also be noted, however, that at both 0 - and $200-\mathrm{msec}$ time lags, there was a sizable positive correlation between head movement and optical movement. In the case of the motion properties of position and velocity, the zero time-lag correlations are higher than they were in the horizontal exafference condition of Experiment 1 .

The changes in visual position constancy registered by the four types of test are shown in Table 4. Only one of the test conditions showed a significant pretestto-posttest change, that with a fixed time lag of $115 \mathrm{msec}$, equal to the mean of the training time lag. An analysis of variance confirmed the differential effect of test conditions. In this analysis, the three factors of test condition, direction of reafference $(+11.5$ or $-11.5 \mathrm{~mm} / \mathrm{deg}$ ), and pretest-posttest were included. The interaction of the last two measures the adapta-

Table 3

Cross-Correlation (r) of Head and Optical Motion in Experiment 2

\begin{tabular}{|c|c|c|c|c|c|c|}
\hline \multirow{3}{*}{$\begin{array}{l}\text { Movement } \\
\text { Property }\end{array}$} & \multicolumn{6}{|c|}{ Time Lag (in Milliseconds) } \\
\hline & \multicolumn{2}{|c|}{0} & \multicolumn{2}{|c|}{100} & \multicolumn{2}{|c|}{200} \\
\hline & $\mathrm{r}$ & \pm & $\mathrm{r}$ & \pm & $r$ & \pm \\
\hline Position & .84 & .01 & .96 & .00 & .92 & .02 \\
\hline Velocity & .69 & .05 & .95 & .03 & .77 & .02 \\
\hline Acceleration & .31 & .06 & .45 & .06 & .30 & .00 \\
\hline
\end{tabular}

Note-Correlations are based on 9-sec samples taken at the beginning of training from all 32 subjects. Positive correlations for a subject are taken as those corresponding to the optical movement: head movement relation of his training condition. $\pm=99 \%$ confidence limits.
Table 4

Position Constance Judgments in Experiment 2

\begin{tabular}{|c|c|c|c|c|c|c|}
\hline \multirow{2}{*}{$\begin{array}{l}\text { Time Lag } \\
\text { in Test }\end{array}$} & \multicolumn{2}{|c|}{ Pretest } & \multicolumn{2}{|c|}{ Posttest } & \multicolumn{2}{|c|}{ Change in Mean } \\
\hline & Mean & \pm & Mean & \pm & Change & \pm \\
\hline 115 & -.04 & .25 & +.51 & .35 & +.55 & .26 \\
\hline 5 & -.06 & .53 & -.15 & .67 & -.09 & .58 \\
\hline 225 & +.16 & .55 & +.42 & .31 & +.26 & .35 \\
\hline $5-225$ & +.02 & .18 & +.22 & .22 & +.19 & .27 \\
\hline
\end{tabular}

Note-Time lags are given in milliseconds. The measures are in terms of the amount of horizontal optical motion (in millimeters) per degree of vertical head motion, at the designated time lag between head motion and optical motion. Positive values indicate optical motion in the direction of that during training. The values are based on 10 pretests and 10 posttests for each subject, there being eight different subjects in each test condition. $\pm=99 \%$ confidence limits.

Table 5

Variability of Position Constancy Judgments in Experiment 2

\begin{tabular}{|c|c|c|c|c|c|c|}
\hline \multirow{2}{*}{$\begin{array}{l}\text { Time Lag } \\
\text { in Test }\end{array}$} & \multicolumn{2}{|c|}{ Pretest } & \multicolumn{2}{|c|}{ Posttest } & \multicolumn{2}{|c|}{ Change in SD } \\
\hline & SD & \pm & SD & \pm & Change & \pm \\
\hline 115 & .51 & .28 & .53 & .24 & .02 & .27 \\
\hline 5 & .55 & .29 & .58 & .34 & .03 & .20 \\
\hline 225 & .35 & .17 & .53 & .37 & .18 & .26 \\
\hline $5-225$ & .28 & .07 & .42 & .21 & .14 & .18 \\
\hline
\end{tabular}

Note-Time lags are given in milliseconds. The measures are in terms of the amount of horizontal optical motion (in millimeters) per degree of vertical head motion, for the indicated time lag between head movement and optical movement. They are based on 10 pretests and 10 posttests for each subject, there being eight different subjects in each of the four test conditions. $\pm=99 \%$ confidence limits.

tion effect, and for this $[F(1,24)=17.642696, p<.001]$. This effect depended on test condition: The triple interaction showed $[F(3,24)=5.851804, p<.005]$. The only other significant effect was the main effect of the direction of reafference $[F(1,24)=6.86832$, $\mathrm{p}<.05]$.

The variability of position constancy judgments is shown in Table 5. None of the pretest-posttest changes is significant, and an analysis of variance showed no statistically significant effects of test condition, direction of reafference, pretest-posttest, or their interactions.

\section{Discussion}

All four groups in this experiment received the same reafference training; they should therefore have been in the same adaptation state at the time of the posttest, with individual differences randomized among groups. The different test conditions led to different pretest-posttest changes, with only one showing a significant change.

This result can be interpreted in terms of the dual 
adaptation state properties of expected optical motion (EOM) and expected time lag (ETL) found by Hay and Goldsmith (1973). Each test condition allowed the subject to adjust only the optical-motion component: The time-lag component varied between conditions. Hence, an optimum match of stimulus conditions during test and the adaptation state could be obtained in only one test condition.

Examination of the results in Table 4 indicates that the maximum, and only reliable, adaptation was in Test Condition 1, in which the time lag was fixed with a value equal to the mean of the training time lag, and corresponded to the lag of the maximum cross-correlation of HM and OM (Table 3). This test condition's time lag, $115 \mathrm{msec}$, is thus the best estimate of the ETL property of the adaptation state.

It should be noted that the adaptation effect for the test whose time lag matched the average lag of training here is closely comparable to that found by Hay and Goldsmith (1973) when the test time lag matched the fixed lag of training. Here, the pretestposttest change was $+.55 \pm .26 \mathrm{~mm} / \mathrm{deg}$; in Experiment 1 of the earlier study, the pretest-posttest change was $+.59 \pm .44 \mathrm{~mm} / \mathrm{deg}$. This suggests that the exafferent time-lag component had no effect on the strength of reafference learning.

\section{GENERAL DISCUSSION}

Visual position constancy adaptation was found to occur in the presence of exafference in optical motion or in the time lag between head motion and optical motion. This suggests a revision of the model for this type of reafference learning advanced by Hay and Goldsmith (1973). The model was derived from Held's (1961) general theory of reafference learning, which uses a kind of associative memory for pairing motor and sensory signals. In Held's associative memory, exafference must cause a variation in the sensory associates of a given motor signal and hence an increase in the variable error of perception. Held and Freedman (1963) report evidence of this effect of exafference in the adaptation of a perceptual skill different from visual position constancy: eye-hand coordination. The absence of such an effect in the case of visual position constancy (cf. Tables 2 and 5) leads us to replace the associative memory with something else for the model of position constancy adaptation.

In place of the above type of associative memory, a statistical correlator that stores, not the "raw data" of motor-sensory pairings, but the variances and covariances of motor and sensory events, is proposed. These statistics are used by the correlator to generate for each head movement (HM) an ex- pected optical movement (EOM), using the following relationship:

$$
\mathrm{EOM}=\mathrm{HM} \frac{\text { covariance }(\mathrm{HM}, \mathrm{OM})}{\text { variance }(\mathrm{HM})} .
$$

The variance of OM does not enter this relationship, and therefore exafference does not alter EOM.

This use of a statistical correlator rather than an associative memory for the model of position constancy, as distinct from eye-hand coordination, is consistent with the different natures of the two skills. In eye-hand coordination, we have a situation in which the viewed object (the hand) seldom moves without the subject's active participation. In visual position constancy, by way of contrast, the viewed object may often undergo subject-independent motion. In the latter case, the only prediction possible is a statistical one. The best such prediction would be based on variance and covariance measures over a range of motor-sensory experiences, rather than "selection from storage ... from among many trace combinations of roughly equal weight" as in the associative memory model (Held, 1961, p. 31).

In the statistical correlator model, the correlation coefficients between HM and OM during training indicate the potential accuracy of the OM prediction. In Experiment 2, evidence is found that the statistical correlator is able to identify the time lag between HM and OM for which the correlation coefficient is maximum, and the adaptation is specific to that time lag.

\section{Relevance of the Present Findings to the Oculomotor Theory of Position Constancy Adaptation}

Hay (1968) found that the type of position constancy adaptation studied here depends on the subject's following the luminous spot with his eyes, and he therefore proposed that the adaptation involves the learning of new compensatory eye movements in response to head movement. Subsequent research on the vestibuloocular reflex (VOR) in humans and animals exposed to inverting spectacles and other novel viewing conditions indicates that such learning can occur (Wilson \& Melvill Jones, 1979). Changes in the timing as well as the magnitude of the VOR have been found, corresponding to the expected time lag and expected optical motion in visual position constancy (Gonshor \& Melvill Jones, 1976; Miles, Braitmand, \& Dow, 1980). The present findings predict that, if this parallel corresponds to an underlying unity, VOR adaptation should occur even when there is a variable (exafferent) component in the training relation between head movement and eye movement. 


\section{REFERENCES}

Gonshor, A., \& Melvill Jones, G. Extreme vestibulo-ocular adaptation induced by prolonged optical reversal of vision. Journal of Physiology, 1976, 256, 381-414.

HAY, J. C. Visual adaptation to an altered correlation between eye movement and head movement. Science, 1968, 160, 429-430.

Hay, J. C. Motor-transformation learning. Perception, 1974, 3, 487-496.

H^y, J. C., \& Goldsmith, W. M. Space-time adaptation of visual position constancy. Journal of Experimental Psychology, 1973, 99, 1-9.

HELD, R. M. Exposure-history as a factor in maintaining stability of perception and coordination. Journal of Nervous and Mental Diseases, 1961, 132, 26-32.

Held, R. M., \& Freedman, S. J. Plasticity in human sensorimotor control. Science, 1963, 142, 455-462.
Holst, E. von. Relations between the central nervous and the peripheral organs, British Journal of Animal Bel 1954, 2, 89-94.

Miles, F. A., Braitman, D. J., \& Dow, B. M. Lo adaptive changes in primate vestibularocular reflex: $\mathrm{I}$. Be observations. Journal of Neurophysiology, 1980, 43, 147

Shebilske, W. L. Visuomotor coordination in visual $d$ and position constancies. In W. Epstein (Ed.), Stabi constancy in visual perception. New York: Wiley, 1977.

WELCH, R. B. Perceptual modification: Adapting to altered environments. New York: Academic Press, 1978.

Wilson, V. J., \& Melvill Jones, G. Mammalian ve physiology. New York: Plenum Press, 1979.

(Manuscript received January 9, 1981; revision accepted for publication June 16, 1981.) 